also capped, delaying treatment for the thousands on waiting lists. Increasing this capacity for vaccinated patients will reduce waiting times and consequentially increase dental access. Similarly, in primary care, increased access will help serve and reduce the great needs of our population.

S. Radia, Bristol, UK https://doi.org/10.1038/s41415-021-2745-1

\section{Vaccination side effects}

Sir, all COVID-19 vaccines aim to actively induce systemic immunisation, yet the possibility of side effects' (SEs') occurrence within the oro-maxillofacial region cannot be ignored. Data collected from worldwide clinical trials carried out in 2020 reported mild to moderate incidence of enlarged lymph nodes (uncommon, 1/100 recipients), sore throat (common, 1/10), and even acute peripheral unilateral facial palsy (rare 1/1,000). ${ }^{1,2}$

The utilisation of Yellow Cards by both professionals and patients, an online system governed by Medications and Healthcare products Regulatory Agency (MHRA), would strongly support the COVID-19 vaccination programmes' clinical safety.

Current evidence is inadequate to accept or reject any causal relationship between COVID-19 vaccinations and adverse events or reactive response affecting the oro-facial region. Although serious orally manifesting SEs are deemed unlikely, the collaborative multicentre cross-sectional survey commenced in January this year is designed to estimate the prevalence of the oral side effects of COVID-19 vaccine and identify risk factors of COVID-19 vaccine adverse events. ${ }^{3}$

While it is our professional duty to act as ambassadors of any vaccination programmes, equally we need to be constantly vigilant and take appropriate action when encountering abnormal orofacial symptoms of unknown origin.

A. Dziedzic, M. Tanasiewicz, Silesia, Katowice, Poland

\section{References}

1. Medicine \& Healthcare products Regulatory Agency. Decision: Information for Healthcare Professionals on COVID-19 Vaccine AstraZeneca. Updated 28 January 2021. Available at: https://www.gov.uk/government/ publications/regulatory-approval-of-covid-19-vaccineastrazeneca/information-for-healthcare-professionals-oncovid-19-vaccine-astrazeneca (accessed 5 February 2021).
2. Medicine \& Healthcare products Regulatory Agency. Decision: Information for Healthcare Professionals on Pfizer/BioNTech COVID-19 vaccine. Updated 28 January 2021. Available at: https://www.gov.uk/government/ publications/regulatory-approval-of-pfizer-biontechvaccine-for-covid-19/information-for-healthcareprofessionals-on-pfizerbiontech-covid-19-vaccine (accessed 5 February 2021).

3. Oral Side Effects of COVID-19 Vaccine. ClinicalTrials. gov Identifier: NCT04706156. U.S. National Library of Medicine. Available at: https://clinicaltrials.gov/ct2/ show/NCT04706156 (accessed 5 February 2021).

https://doi.org/10.1038/s41415-021-2746-0

\section{Fit testing troubles?}

Sir, the use of $3 \mathrm{D}$ face scanning in dentistry is nothing new; clinicians and dental laboratories use this technology as an integral part of diagnostic, surgical and restorative treatment planning. During the COVID19 pandemic, this technique is being used to produce a bespoke frame to achieve a facial seal for disposable FFP3 masks, in circumstances when achieving a seal has proved challenging. Being unable to 'fit test' dental clinicians despite trying various makes and models of FFP3 masks was the driver to explore this solution.

We used the Bellus3D face-scanning app, downloaded from the App Store. The 'Mask Fitter' option was selected to produce a 3D scan of the face. An STL file for laboratory printing produced a standard frame (Fig. 1) which fits over the FFP3 mask to produce a tight facial seal. The frame is held in place by attached elastic straps (Fig. 2). This technique

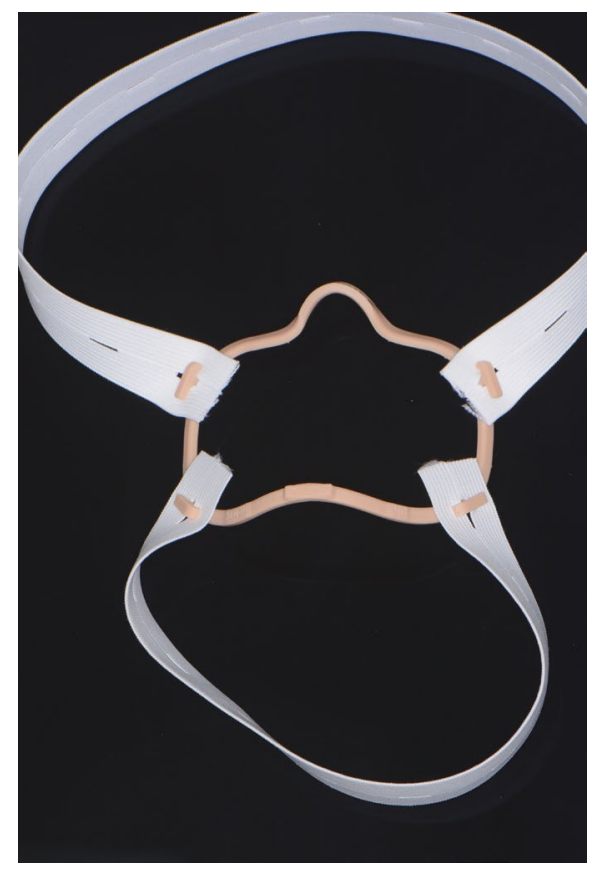

Fig. 1 Standard frame produced by an STL file has resulted in individuals subsequently achieving a passed fit test for a FFP3 mask which has previously failed to produce an adequate seal on its own.

This is a cost-effective option when compared to purchasing a powered air purifying respirator (PAPR) which would be the only other alternative. The total cost of this face-scanning solution including purchasing the app, STL files, laboratory fees and elastics was around $£ 25$. Simple steps to follow:

1. Download the app https://apps. apple.com/us/app/bellus3d-faceapp/ id1352268131

2. Open the app and place your face in the red demarcated area

3. This produces a 3D scan of the face. Choose the 'Mask Fitter' option on the menu bar

4. Choose frame type

5. Choose Export (unless you have your own $3 \mathrm{D}$ printer)

6. Pay the Export fee through the App Store

7. Email created STL files to the laboratory or $3 \mathrm{D}$ printing centre

8. Purchase elastic roll with eyelets to fit the frame to the face

9. Conduct fit test with FFP3 mask and frame in place.

K. Shah, D. Bentley, Hartlepool, UK https://doi.org/10.1038/s41415-021-2747-z

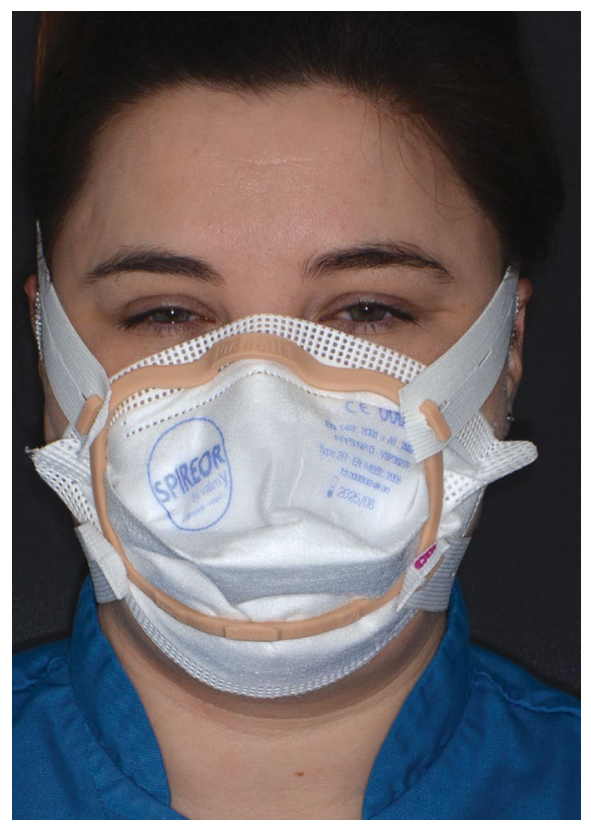

Fig. 2 The frame is held in place by attached elastic straps 